\title{
PRIMARY BILATERAL BREAST LYMPHOMA: A REVIEW OF LITERATURE AND REPORT OF FOUR CASES FROM A SINGLE CENTRE
}

\author{
Razia Bano ${ }^{1}$, Amina Iqbal Khan², Amna Shahraf ${ }^{3}$, Mahwish Abid ${ }^{1}$ \\ ${ }^{1}$ Department of Surgery, Combined Military Hospital Rawalpindi, Rawalpindi, Pakistan, ${ }^{2}$ Department of Surgical \\ Oncology, Shaukat Khanum Memorial Cancer Hospital and Research Centre, Lahore, Pakistan, ${ }^{3}$ Department of \\ Anaesthesia, Combined Military Hospital Rawalpindi, Rawalpindi, Pakistan
}

Received: 17 January 2016 / Accepted: 15 July 2016

\begin{abstract}
Primary breast lymphoma is a rare entity and carries poor prognosis, bilateral breast lymphoma is even rarer and carries worst prognosis. Bilateral breast lymphoma is a rare disease and lacks treatment. Out of the 2766 cases of nonHodgkin's lymphoma registered at our institute from 1994 to 2013, 31 cases of breast lymphoma were found, of which four cases had bilateral involvement. In this review, we describe clinical presentation, histopathological subtypes, treatment administered and outcome of those four cases retrospectively. All patients were female with a median age of 31 years (range 24-64 years). Three patients were diagnosed with diffuse large B-cell lymphoma and mucosaassociated lymphoid tissue lymphoma detected in one patient. Chemotherapy remained the main treatment modality and surgery (excision biopsy) was reserved for diagnostic purpose only, none of the patients received radiation therapy.
\end{abstract}

Key words: Breast lymphoma, histopathological subtypes, palliation

\section{Introduction}

Primary breast lymphoma (PBL) is a rare form of breast malignancy and accounting $0.4-0.5 \%$ of all breast cancers and $1.7-2.2 \%$ of extranodal non-Hodgkin's lymphoma (NHL). ${ }^{[1]}$ PBLs are classified as disease confined to the breast only in the absence of previously existing lymphoma. ${ }^{[2]}$ According to Wiseman and Liao criteria, PBL is defined by the presence of following features: (1) Breast should be the primary site, (2) there should be no evidence of the distant organ involvement, (3) tissue sample should be adequate and (4) there should be a close association between breast tissue and lymphoma. ${ }^{[3]}$ According to this definition, lymphoma confined to the breast alone will be classified as PBL. However, some researchers would also category PBL when bone marrow is involved in addition to lymphoma in the breast. ${ }^{[4]}$ Bilateral breast lymphoma accounts for about $11 \%$ of lymphoma involving the breast, whereas a study of 204 primary diffuse large B-cell lymphoma (DLBCL) patients by Ryan et al. showed bilateral breast involvement by lymphoma in only $5 \%$ of cases. ${ }^{[5,6]}$ Cancer registry

Correspondence: Dr. Razia Bano, Department of Surgery, Combined Military Hospital Rawalpindi, Rawalpindi, Pakistan.

Email: raazia240@gmail.com at Shaukat Khanum Memorial Cancer Hospital and Research Centre (SKMCH and RC), Lahore, recorded 12,866 cases of breast cancer between December 1994 and December 2013. Amongst those patients, four cases of PBL were identified whose details are given in this case report.

\section{Materials and Methods}

Data on four PBL patients treated at SKMCH and RC were collected by review of their case notes using hospital electronic medical records in a retrospective manner. Following patient variables searched: Patients' demographics such as age and gender, presenting clinical symptoms such as B-symptoms and pathological characteristics such as histopathology and immunohistochemistry of breast biopsy as well as bone marrow infiltration and its response to chemotherapy if positive at baseline, details of treatment and measurement of outcome. A formal ethical approval was obtained from the institutional review board.

\section{Case Series}

Baseline patient characteristics are shown in Table 1. All patients were female, median age at the time of diagnosis 
was 31 years (range 24-64 years). All patients underwent breast ultrasound, mammogram, bone marrow biopsy and whole-body computed tomography for staging of lymphoma at presentation. Diagnosis was made on review of the blocks as all four patients had undergone lumpectomy at peripheral hospitals before being referred to our institute for further. Ann Arbor system was used for staging of lymphoma according to which bilateral cases were classified as Stage IVE due to poor prognosis and according to Wiseman and Liao criteria. ${ }^{[3]}$

At presentation, one patient has multiple bilateral skin nodules and two were diagnosed with pregnancy/ lactation-associated breast lumps. Two of our patients had B symptoms and another two had bone marrow involvement at baseline. Histopathological diagnosis of DLBCL was made in three patients and one case had morphology consistent with mucosa-associated lymphoid tissue (MALT) lymphoma. Immunohistochemistry consistent with the given diagnosis was the positivity of CD 20, CD 30 and Ki 67 in three cases; however, immunohistochemistry was unknown for a single patient. There was no distant organ involvement at the time of presentation in all four cases.

The main stay of treatment was R-CHOP chemotherapy (rituximab, cyclophosphamide, doxorubicin, vincristine and prednisolone). Surgery was limited for diagnostic purpose only, none of our patients received radiation therapy to breast. Response to the treatment was assessed by bone marrow biopsy after chemotherapy and PET scan. One patient died during the chemotherapy and the second patient had complete response, while rest of the two patients had progressive disease after chemotherapy, none of the patient received extensive surgery or radiation therapy, survival period ranges from 1 to 13 months; three patients died and two were lymphoma specific deaths [Table 2].

\section{Discussion}

PBL is a rare entity, it represents $0.04-0.5 \%$ of all breast malignancies. ${ }^{[6]}$ Breast lymphomas are staged according to Ann Arbor staging system, although staging of bilateral PBL is still controversial, Ryan et al. classified bilateral PBL in Stage IVE, ${ }^{[5]}$ this was the largest study of PB-DLBCL; they reviewed retrospectively 204 patients, whereas other studies include in Stage IE and IIE. ${ }^{[7,8]} \mathrm{We}$ included bilateral involvement in Stage IVE as they had poor prognosis. Bilateral PBLs have some association with pregnancy as mentioned in some studies. ${ }^{[9]}$ It is difficult to differentiate radiologically between breast lymphoma, fibroadenoma and breast cancer. ${ }^{[1,10]}$

The most common subtype is DLBCL accounts for about $50 \%$ of all BCLs, other rare types include MALT lymphoma, Burkitt lymphoma, anaplastic and follicular, small lymphocytic. ${ }^{[11]}$ Aggressive form of PBL is known

Table 1: Demographics

\begin{tabular}{l|l|l|l|l|l|l}
\hline Case & Age & Diagnosis & B symptoms & $\begin{array}{l}\text { Pre-treatment } \\
\text { bone marrow }\end{array}$ & $\begin{array}{l}\text { Post-treatment } \\
\text { bone marrow }\end{array}$ & Risks \\
\hline 1 & 27 & DLBCL & No & Positive & Negative & Pregnancy associated \\
\hline 2 & 35 & DLBCL & No & Negative & Negative & Multiple skin lesions \\
\hline 3 & 64 & MALT lymphoma & Yes & Positive & Positive & None \\
\hline 4 & 24 & DLBCL & Yes & Negative & Negative & Lactation associated \\
\hline \multicolumn{7}{l}{ MALT: Mucosa-associated lymphoid tissue, DLBCL: Diffuse large B-cell lymphoma }
\end{tabular}

Table 2: Treatment and outcome

\begin{tabular}{l|l|l|l|l|l|l}
\hline Case & Chemotherapy & Radiotherapy & Response & Local relapse & Distant relapse & Outcome \\
\hline 1 & R-CHOP X 6 & No & PR & No & No & Dead \\
\hline 2 & R-CHOP X 6 & No & CR & No & No & Dead \\
\hline 3 & R-CHOP X 5 & No & Progressive & No & No & Dead \\
\hline 4 & R-CHOP X 4, RICE X 5 & No & Progressive & No & No & Alive \\
\hline
\end{tabular}


to have association with younger age group; three of our patients were young. ${ }^{[12]}$

Another largest study by Jeanneret-Sozzi et al. included a review of 84 cases of PBL from 20 institutions, they concluded that PBL has better prognosis if managed by combined chemotherapy and radiotherapy. ${ }^{[13]}$ MALT lymphoma breast is an extremely rare entity amongst the NHL, and bilateral involvement is even rarer, we found only single patient with bilateral MALT lymphoma. ${ }^{[14]}$

Chemotherapy stays the main treatment modality for breast lymphomas; R-CHOP is the preferred regime, as it increases 5-year survival. Aviv et al. recommended addition of Rituximab to chemotherapy may reduce central nervous system relapse and may be given intrathecal injections, ${ }^{[2,15]}$ whereas other studies describe it as ineffective. ${ }^{[16]}$ Radiotherapy can be combined with chemotherapy, but the role of surgery is limited only to diagnosis and palliation of symptoms in progressive disease. Jennings et al. observed high mortality rate in patients undergoing mastectomy and axillary dissection; therefore, they concluded that there is no role of axillary lymph nodal dissection and extensive surgery for breast lymphoma. ${ }^{[17,18]}$

\section{Conclusion}

Bilateral breast lymphoma is very rare, but aggressive disease with poor prognosis usually present at younger age group, aggressive treatment with chemotherapy should be considered and surgery should be reserved for diagnosis and palliation.

\section{Acknowledgment}

We are thankful to Dr. Sajid Mushtaq, Misbah (Pathology Department), and Dr. Farhana Badar and Mr. Shahid Mehmood (Cancer Registry Department) for helping us in retrieving the data of breast lymphoma.

\section{Conflict of Interest}

The authors declare that they have no conflict of interest.

\section{References}

1. Arber DA, Simpson JF, Weiss LM, et al. Non-Hodgkin's lymphoma involving the breast. Am J Surg Pathol
1994;18:288-95.

2. Jeanneret-Sozzi W, Taghian A, Epelbaum R, et al. Primary breast lymphoma: Patient profile, outcome and prognostic factors. A multicentre rare cancer network study. BMC Cancer 2008;8:86.

3. Wiseman C, Liao KT. Primary lymphoma of the breast. Cancer 1972;29:1705-12.

4. Brustein S, Filippa DA, Kimmel M, et al. Malignant lymphoma of the breast. A study of 53 patients. Ann Surg 1987;205:144-50.

5. Ganjoo K, Advani R, Mariappan MR, et al. Non-Hodgkin lymphoma of the breast. Cancer 2007;110:25-30.

6. Ryan G, Martinelli G, Kuper-Hommel M, et al. Primary diffuse large B-cell lymphoma of the breast: Prognostic factors and outcomes of a study by the international extranodal lymphoma study group. Ann Oncol 2008;19:233-41.

7. Dawn B, Perry MC. Bilateral non-Hodgkin's lymphoma of the breast mimicking mastitis. South Med J 1997;90:328-9.

8. Fukuhara S, Watanabe T, Munakata W, et al. Bulky disease has an impact on outcomes in primary diffuse large B-cell lymphoma of the breast: A retrospective analysis at a single institution. Eur J Haematol 2011;87:434-40.

9. Julen O, Dellacasa I, Pelte MF, et al. Primary breast lymphomas. Rare Tumors 2009;1:e14.

10. Jeon HJ, Akagi T, Hoshida Y, et al. Primary non-hodgkin malignant lymphoma of the breast. An immunohistochemical study of seven patients and literature review of 152 patients with breast lymphoma in Japan. Cancer 1992;70:2451-9.

11. Sabaté JM, Gómez A, Torrubia S, et al. Lymphoma of the breast: Clinical and radiologic features with pathologic correlation in 28 patients. Breast J 2002;8:294-304.

12. Joks M, Myśliwiec K, Lewandowski K. Primary breast lymphoma-a review of the literature and report of three cases. Arch Med Sci 2011;7:27-33.

13. Brogi E, Harris NL. Lymphomas of the breast: Pathology and clinical behavior. Semin Oncol 1999;26:357-64.

14. Gopal S, Awasthi S, Elghetany MT. Bilateral breast MALT lymphoma: A case report and review of the literature. Ann Hematol 2000;79:86-9.

15. Aviv A, Tadmor T, Polliack A. Primary diffuse large B-cell lymphoma of the breast: Looking at pathogenesis, clinical issues and therapeutic options. Ann Oncol 2013;24:2236-44.

16. Hosein PJ, Maragulia JC, Salzberg MP, et al. A multicentre study of primary breast diffuse large B-cell lymphoma in the rituximab era. Br J Haematol 2014;165:358-63.

17. Validire P, Capovilla M, Asselain B, et al. Primary breast non-Hodgkin's lymphoma: A large single center study of initial characteristics, natural history, and prognostic factors. Am J Hematol 2009;84:133-9.

18. Jennings WC, Baker RS, Murray SS, et al. Primary breast lymphoma: The role of mastectomy and the importance of lymph node status. Ann Surg 2007;245:784-9. 